\title{
Anharmonic phonon-phonon scattering for modeling of three-dimensional atomistic transport: an original quantum treatment
}

\author{
Y. Lee ${ }^{1, *}$ M. Bescond,${ }^{2}{ }^{\dagger}$ D. Logoteta,${ }^{3}$ N. Cavassilas,${ }^{3}$ M. Lannoo, ${ }^{3}$ and M. Luisier ${ }^{1}$ \\ ${ }^{1}$ Integrated Systems Laboratory, ETH Zürich, 8092 Zürich, Switzerland \\ ${ }^{2}$ LIMMS, CNRS-Institute of Industrial Science, UMI 2820, University of Tokyo, 153-8505 Tokyo, Japan \\ ${ }^{3}$ IM2NP, UMR CNRS 7334, Aix-Marseille Université, Technopôle de Château-Gombert, \\ Bâtiment Néel, 60 Rue Frédéric Joliot Curie, 13453 Marseille, France
}

\begin{abstract}
We propose an original method to quantum mechanically treat anharmonic interactions in the atomistic non-equilibrium Green's function (NEGF) simulation of phonon transport. We demonstrate that the so-called lowest order approximation (LOA), implemented through a rescaling technique and analytically continued by means of the Padé approximants, can be used to accurately model a third-order anharmonicity. Although the paper focuses on a specific self-energy, the method is applicable to a very wide class of physical interactions. We apply this approach to the simulation of the anharmonic phonon transport in realistic Si and Ge nanowires with uniform or discontinuous cross-section. The effect of increasing the temperature above $300 \mathrm{~K}$ is also investigated. In all the considered cases, we were able to obtain a good agreement with the routinely adopted self-consistent Born approximation (SCBA), at a remarkably lower computational cost. In the more complicated case of temperatures much higher than the room temperature, we found that the first-order Richardson extrapolation applied to the sequence of the Padé approximants $\mathrm{N}-1 / \mathrm{N}$ results in a significant acceleration of the convergence.
\end{abstract}

\section{INTRODUCTION}

The semiconductor fabrication technology exhibits nowadays the capability of manipulating materials down to the atomic level, therefore offering the opportunity to realize well-defined nanoscale structures ${ }^{1-5}$. Nanoscale systems are very attractive, since their use has been recognized as a powerful option to promote technology advances in several different application fields, ranging from nanoelectronics (e.g. transistors $^{6,7}$ and tunnel diodes ${ }^{8}$ ) and nanophotonics (e.g. photovoltaics ${ }^{9,10}$ ) to energy harvesting devices (e.g. thermoelectric devices ${ }^{11,12}$ ).

The development of nanoscale devices requires the modeling, on a quantum mechanical basis, of the physics underlying the transport processes. Large efforts have been traditionally devoted to investigate and accurately describe electron transport mechanisms ${ }^{13-19}$. More recently, however, an increasing number of researches ${ }^{20-25}$ have emphasized the fundamental role played by thermal effects at the nanometric scale. Self-heating, for example, has been shown to considerably reduce performance ${ }^{26}$ and lifetime ${ }^{27}$ of nanoscale electronic and optoelectronic devices. Furthermore, nanostructures have been recognized as promising building blocks for thermoelectric applications, due to their small thermal conductivity ${ }^{28}$. Despite this increasing interest, the understanding of nanoscale quantum phonon transport is still lacking compared to the electron one, and further studies are needed to gain a detailed physical picture, particularly for what concerns the role of anharmonic phonon-phonon interactions.

Nanoscale thermal transport has been investigated by adopting several approaches (e.g. the linearized Boltzmann transport equation based on the Fermi golden rule $^{22,29-32}$, (non)-equilibrium molecular dynamics ${ }^{33-37}$ and first-principles perturbation theory ${ }^{38-40}$ ), able to capture quantum mechanical effects within different degrees of approximation. Among them, the nonequilibrium Green's function (NEGF) framework has attracted wide interest, as it offers the opportunity to perform full quantum simulations, including many-body effects (e.g. anharmonic phonon interactions ${ }^{20,21,25,41,42}$ ) via suitable self-energies.

From the numerical point of view, the main bottleneck in applying the NEGF formalism, usually comes from the iterative solution within the self-consistent Born approximation (SCBA) of the non-linear Dyson equation. This task, particularly heavy in the simulation of threedimensional realistic nanostructures, requires performing a large number of iterations in order to obtain accurate results for the physical quantities of interest. This is partly due to the intrinsic convergence properties of the SCBA iterative scheme, which provides a self-energy fulfilling the conservation laws (in particular, a $\Phi$-derivable self-energy ${ }^{43-46}$ ) only asymptotically.

In the last decade, several works, focusing on the modeling of the electron-phonon interaction, have investigated the possibility of avoiding such an expensive iterative scheme ${ }^{47-54}$. In this paper, we tackle this problem by considering a different kind of self-energy, which describes the dominant phonon-phonon anharmonic interaction ${ }^{41}$ and is not a linear functional with respect to the phonon Green's function. We will show that the so-called Lowest Order Approximation (LOA) ${ }^{49,50,52,54}$, combined with a rescaling technique and the Padé approximation, can also be effectively used in this case to compute the chargeconserving expectation values of a generic linear operator at any order. Importantly, the specific case considered turns out to be just an example of a very wide class of self-energies to which the method is applicable.

In order to assess the numerical efficiency of the method against the SCBA, we simulated the anharmonic 
phonon transport in $\mathrm{Si}$ and Ge nanowires (NWs). They are a relevant example of $3 \mathrm{D}$ nanostructures with potentialities in a variety of application fields where the thermal transport plays a relevant role and needs to be accurately modeled. Several configurations have been considered, differing in the NW crystallographic orientation, the value of the temperature and the regularity of the NW surface. The atomistic simulations have been carried out within the NEGF framework by using the $\mathrm{OMEN}^{25}$ code, which implements a modified-valenceforce field (VFF) model to describe the interatomic potential.

As key findings, we report that for all the considered structures at room temperature the third-order thermal currents obtained from LOA diagrams, with an analytical continuation of Padé approximants, can reconstruct the SCBA values and lighten the SCBA computational burden. For anharmonic effects at high temperature (500 $\mathrm{K}-900 \mathrm{~K}$ ), it turns out that higher-order LOA thermal currents are required. In such a case, we suggest as a solution the application of the first-order Richardson extrapolation $^{55-57}$ to the sequence of the Padé approximants $N-1 / N$.

The rest of the paper is organized as follows. In Sec. II, we first describe the NEGF model for the anharmonic phonon transport. We then introduce the LOA rescaling technique for the case of the considered phononphonon interaction self-energy, and the application of the Richardson extrapolation to the Padé approximants $N-1 / N$. In Section III, we present the simulation results obtained within the LOA and systematically compare them with the corresponding obtained within the SCBA. Conclusions and perspective for future developments are followed in Sec. IV.

\section{THEORY}

\section{A. Phonon transport model}

The phonon Hamiltonian in three dimensions reads as

$$
\begin{aligned}
\mathbf{H}_{\mathrm{ph}}= & \sum_{l_{1}} \sum_{\mu} \frac{\hat{P}_{\mu}^{2}}{2 M_{\mu}}+\frac{1}{2} \sum_{l_{1}, l_{2}} \sum_{\mu, \nu} \hat{u}_{\mu}^{l_{1}} \hat{u}_{\nu}^{l_{2}} \cdot \frac{\partial^{2}}{\partial R_{\mu}^{l_{1}} \partial R_{\nu}^{l_{2}}} V_{\mathrm{harm}} \\
& +\frac{1}{3 !} \sum_{l_{1}, l_{2}, l_{3}} \sum_{\mu, \nu, \sigma} \hat{u}_{\mu}^{l_{1}} \hat{u}_{\nu}^{l_{2}} \hat{u}_{\sigma}^{l_{3}} \cdot \frac{\partial^{3}}{\partial R_{\mu}^{l_{1}} \partial R_{\nu}^{l_{2}} \partial R_{\sigma}^{l_{3}}} V_{\text {anh }} \\
& +O\left(\hat{u}_{\mu}^{4}\right),
\end{aligned}
$$

where the first, second, and third term refers to the kinetic, harmonic, and anharmonic contributions, respectively. The indices $\mu, \nu$, and $\sigma$ denote the atomic postions, while the indices $l_{1}, l_{2}$, and $l_{3}$ run over the $\mathrm{x}$, y, and z directions. $\mathbf{R}_{\mu}, M_{\mu}$, and $\hat{P}_{\mu}$ are the position, the mass, and the momentum operator, respectively, of the atom at the position $\mu$. The operator $\hat{u}_{\mu}^{l_{1}}$ is the quantized displacement of the atom at the position $\mu$ from the equilibrium position along the direction $l_{1}$. In this work, $V_{\text {harm }}$ and $V_{\text {anh }}$ are the VFF harmonic ${ }^{58,59}$ and anharmonic ${ }^{25}$ potential energies, respectively. Free boundary conditions are enforced on the external surface of the nanowire.

The second derivative of the VFF harmonic potential energy with respect to the atomic position $\mu$ in the $l_{1}$ direction and to the atomic position $\nu$ in the $l_{2}$ direction corresponds to the entries of the dynamical matrix $\boldsymbol{\Phi}$

$$
\Phi_{\mu \nu}^{l_{1} l_{2}}=\frac{\partial^{2}}{\partial R_{\mu}^{l_{1}} \partial R_{\nu}^{l_{2}}} V_{\text {harm }} .
$$

The retarded $\left(D^{R}(\omega)\right)$ and greater/lesser $\left(D^{\gtrless}(\omega)\right)$ phonon Green's functions satisfy the equations:

$$
\begin{gathered}
\sum_{l_{1}} \sum_{\mu}\left[M_{\nu} \omega^{2} \delta_{l_{2} l_{1}} \delta_{\nu \mu}-\Phi_{\nu \mu}^{l_{2} l_{1}}-\Pi_{\nu \mu}^{R B, l_{2} l_{1}}(\omega)-\Pi_{\nu \mu}^{R S, l_{2} l_{1}}(\omega)\right] D_{\mu \sigma}^{R, l_{1} l_{3}}(\omega)=\delta_{l_{2} l_{3}} \delta_{\nu \sigma}, \\
D_{\nu \sigma}^{\gtrless, l_{2} l_{3}}(\omega)=\sum_{l_{1}, l_{1}^{\prime}} \sum_{\mu, \mu^{\prime}} D_{\nu \mu}^{R, l_{2} l_{1}}(\omega)\left(\Pi_{\mu \mu^{\prime}}^{\gtrless B, l_{1} l_{1}^{\prime}}(\omega)+\Pi_{\mu \mu^{\prime}}^{\gtrless S, l_{1} l_{1}^{\prime}}(\omega)\right) D_{\mu^{\prime} \sigma}^{A, l_{1}^{\prime} l_{3}}(\omega)
\end{gathered}
$$

where $D^{A}(\omega)=\left(D^{R}(\omega)\right)^{\dagger}$ is the advanced phonon Green's function, $\omega$ is the phonon frequency, $\Pi^{R B}(\omega)$ $(\Pi \gtrless B(\omega))$ is the retarded (greater/lesser) open boundary self-energy ${ }^{60}$, and $\Pi^{R S}(\omega)\left(\Pi^{\gtrless S}(\omega)\right)$ is the retarded (greater/lesser) anharmonic phonon-phonon scattering self-energy ${ }^{25}$. The kinetic and harmonic terms of the phonon Hamiltonian are therefore directly included in the phonon Green's function, while the anharmonic term is perturbatively treated by defining a scattering selfenergy.

In this work, we model the anharmonic interactions in terms of the lowest order three-phonon process depicted in Fig. 1. It consists in the decay of a high-energy phonon 
$\left(\omega+\omega^{\prime}\right)$ into two lower energy phonons $\left(\omega\right.$ and $\left.\omega^{\prime}\right)$ or vice versa $^{25,61}$. The corresponding greater/lesser self-energy turns out to be $\Phi$-derivable ${ }^{62}$ and reads as ${ }^{25}$ :

$$
\begin{aligned}
\Pi_{\nu \sigma}^{\gtrless S, l_{2} l_{3}}(\omega)= & 2 i \hbar \sum_{l_{1}, l_{1}^{\prime} l_{1}^{\prime \prime}, l_{1}^{\prime \prime \prime}} \sum_{\mu, \mu^{\prime}, \mu^{\prime \prime}, \mu^{\prime \prime \prime}} \int_{-\infty}^{\infty} \frac{d \omega^{\prime}}{2 \pi} d V_{\nu \mu \mu^{\prime}}^{(3) l_{2} l_{1} l_{1}^{\prime}} \\
& \times D_{\mu \mu^{\prime \prime}}^{\gtrless, l_{1} l_{1}^{\prime \prime}}\left(\omega+\omega^{\prime}\right) D_{\mu^{\prime \prime \prime} \mu^{\prime}}^{\lessgtr, l_{1}^{\prime \prime \prime}} l_{1}^{\prime}\left(\omega^{\prime}\right) \\
& \times d V_{\mu^{\prime \prime} \mu^{\prime \prime \prime} \sigma}^{(3) l_{1}^{\prime \prime} l_{1}^{\prime \prime \prime} l_{3}}
\end{aligned}
$$

with

$$
d V_{\nu \mu \mu^{\prime}}^{(3) l_{2} l_{1} l_{1}^{\prime}}=\frac{\partial^{3}}{\partial R_{\nu}^{l_{2}} \partial R_{\mu}^{l_{1}} \partial R_{\mu^{\prime}}^{l_{1}^{\prime}}} V_{\text {anh }}
$$

The retarded scattering self-energy can be derived from the lesser and greater self-energies as

$$
\begin{aligned}
\Pi_{\nu \sigma}^{R S, l_{2} l_{3}}(\omega)= & \frac{1}{2}\left[\Pi_{\nu \sigma}^{>S, l_{2} l_{3}}(\omega)-\Pi_{\nu \sigma}^{<S, l_{2} l_{3}}(\omega)\right] \\
& +i \mathcal{P} \int \frac{d \omega^{\prime}}{2 \pi} \frac{\Pi_{\nu \sigma}^{>S, l_{2} l_{3}}\left(\omega^{\prime}\right)-\Pi_{\nu \sigma}^{<S, l_{2} l_{3}}\left(\omega^{\prime}\right)}{\omega-\omega^{\prime}}
\end{aligned}
$$

where $\mathcal{P}$ is the Cauchy principal integral value. In order to exploit the efficient recursive algorithm of Ref. 63 to solve Eqs. (3), we resort to a diagonal approximation ${ }^{25,54}$ of the scattering self-energy with respect to the atomic position indexes $\nu$ and $\sigma$. Although this approximation may lead to an underestimation of the interaction strength, in our model this is partly compensated through the calibration of the model parameters for the anharmonic force against available experimental data ${ }^{25}$. As a further approximation, we neglect the real part of the retarded self-energy, as it mainly contributes to an energy renormalization ${ }^{64-66}$. Finally, the phonon frequency shift due to the temperature-dependent lattice expansion is not taken into account.

The self-consistent solution of Eqs. (3), (4) and (6) provides the interacting Green's functions within the SCBA. The local thermal current $\mathcal{I}_{\mathrm{ph}}^{p, p+1}$ between two unit cells $p$ and $p+1$ can then be calculated as

$$
\begin{aligned}
\mathcal{I}_{\mathrm{ph}}^{p, p+1}= & \frac{\hbar}{2 \pi} \sum_{\nu \in p} \sum_{\sigma \in p+1} \sum_{l_{2}, l_{3}} \\
& \times \int_{0}^{\infty} d \omega \omega\left[\Phi_{\nu \sigma}^{l_{2} l_{3}} D_{\sigma \nu}^{<, l_{3} l_{2}}-D_{\nu \sigma}^{<, l_{2} l_{3}} \Phi_{\sigma \nu}^{l_{3} l_{2}}\right],
\end{aligned}
$$

where the atoms at the positions $\nu$ and $\sigma$ belong to the unit cells $p$ and $p+1$, respectively. A unit cell is composed of $N$ consecutive atomic layers along the transport direction, with $N=4$ for the $\langle 100\rangle$ direction and $N=2$ for the $\langle 110\rangle$ one.

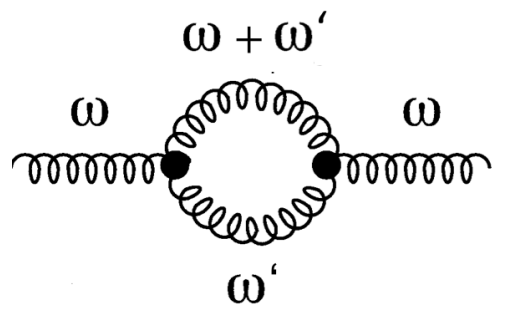

FIG. 1. Lowest order anharmonic phonon-phonon self-energy diagram, describing the anharmonic decay of a high-energy phonon $\left(\omega+\omega^{\prime}\right)$ into two lower energy phonons $\left(\omega\right.$ and $\left.\omega^{\prime}\right)$.

\section{B. Lowest Order Approximation}

The approach routinely used in NEGF numerical simulations to compute an interacting Green's function $D$ consists in iteratively solving, within the SCBA, the Dyson equation ${ }^{13}$

$$
D=d_{0}+d_{0} \Pi[D D] D,
$$

where $d_{0}$ denotes the non-interacting Green's function, $\Pi$ is the self-energy that accounts for the interactions, and the simplified matrix notation $\left[i=\left(r_{i}, \omega_{i}\right), d_{0} \Pi D=\right.$ $\left.\int d 2 \int d 2^{\prime} d_{0}(1 ; 2) \Pi\left(2 ; 2^{\prime}\right) D\left(2^{\prime} ; 1^{\prime}\right)\right]$ has been used. The product $D D$ in the argument of $\Pi$ highlights that the considered self-energy (4) depends on the square of the Green's function. The iteration scheme to solve Eq. (8) can be expressed as

$$
D_{N}=\left[d_{0}^{-1}-\Pi\left[D_{N-1} D_{N-1}\right]\right]^{-1},
$$

where $D_{N}$ denotes the Green's function approximation at the $N^{t h}$ iteration and $D_{0}=d_{0}$. By performing a Taylor series expansion in powers of $\Pi\left[D_{N-1} D_{N-1}\right]$, we can also rewrite Eq. (9) as

$$
\begin{aligned}
D_{N}= & d_{0}+d_{0} \Pi\left[D_{N-1} D_{N-1}\right] d_{0} \\
& +d_{0} \Pi\left[D_{N-1} D_{N-1}\right] d_{0} \Pi\left[D_{N-1} D_{N-1}\right] d_{0}+\cdots .
\end{aligned}
$$

Moreover, by recursively expanding $D_{N-1}$ in terms of $\Pi\left[D_{N-2} D_{N-2}\right], \Pi\left[D_{N-3} D_{N-3}\right], \cdots, \Pi\left[d_{0} d_{0}\right]$, we can rearrange the right hand side of Eq. (10) in the form of products and compositions of the basic term $d_{0} \Pi\left[d_{0} d_{0}\right]$ :

$$
D_{N}=d_{0}+d_{0} \Pi\left[d_{0} d_{0}\right] d_{0}+d_{0} \Pi\left[d_{0} d_{0}\right] d_{0} \Pi\left[d_{0} d_{0}\right] d_{0}+\cdots
$$

The second term on the right hand side of Eq. (11) corresponds to the diagram of Fig. 1, while the other terms 
correspond to higher order diagrams. Here and in the following, the order of a term/diagram is intended as its order in $\Pi$, namely as the number of occurrences of $\Pi$ in that term/diagram. For each $N$, the expansion (11) can be split in two parts: a perturbation series truncated at the order $N$, which includes all the possible (non-crossing) diagrams of order smaller or equal to $N$, and the remaining infinite sum, which misses some diagrams at any order. It can be $\operatorname{shown}^{50}$ that, if the self-energy is $\Phi$-derivable, the perturbation series part always provides a conserving approximation of the Green's function. On the contrary, due to the presence of the renormalizing higher order terms, the SCBA provides a conserving approximation only asymptotically, when the self-consistency is achieved.

Differently from the SCBA, the LOA provides a sequence of expectation values of a generic linear operator by directly relying on the Green's function perturbation series. The $N^{t h}$ element of the sequence, which is referred to as LOA of order $N$, is computed by approximating the Green's function with its perturbation series truncated at the order $N$. According to the considerations above, each element of the sequence fulfills the conservation laws. However, as the perturbation series has a finite convergence radius ${ }^{53}$, the sequence of expectation values can also diverge. In order to turn this sequence into a convergent one, analytical continuation techniques can be resorted.

By using these techniques, higher order effects missed in the truncated perturbation series can be included, therefore obtaining a renormalized approximation of the LOA expectation values. These latter can still fulfill the conservation laws, if the analytical continuation preserves the conserving character of the LOA. Essentially, while both the SCBA and the analytically continued LOA implement a convergent iterative scheme, only the latter provides conserving results at each iteration. In the context of electron-phonon interaction, this difference has been shown to result in a significant acceleration of the convergence of the LOA with respect to the $\mathrm{SCBA}^{52,54}$. In the following, we will show how, by using a rescaling technique, the LOA expectation values can be obtained from the non-conserving estimates provided by the SCBA during the iteration process.

The idea of the rescaling technique is to precondition the expression of $\Pi\left[D_{N-1} D_{N-1}\right]$ in order to obtain, as the result of the SCBA iteration, a "rescaled" version of the expansion (11), in which terms corresponding to diagrams of increasing order are divided by increasing powers of the same scaling factor $\lambda$. A rescaled expression for $D_{N}$, in which terms of order $i$ are divided by $\lambda^{i}$, can be easily obtained by using in the SCBA algorithm a version of $\Pi\left[D_{N-1} D_{N-1}\right]$ in which any occurrence of the functional $\Pi$ is divided by $\lambda$. If $\lambda$ is suitably chosen, the nonconserving terms of order higher than $N$ will be strongly suppressed relative to the conserving ones, thus making it possible to isolate them and build the corresponding LOA. We proceed now to show how the rescaling technique can be implemented in practice, by describing its application to the first two SCBA iterations.

By rescaling $\Pi\left[d_{0} d_{0}\right]$ by $\lambda_{1}$, the first SCBA iteration gives

$$
D_{1}=d_{0}+\frac{d_{0} \Pi\left[d_{0} d_{0}\right] d_{0}}{\lambda_{1}}+\frac{d_{0} \Pi\left[d_{0} d_{0}\right] d_{0} \Pi\left[d_{0} d_{0}\right] d_{0}}{\lambda_{1}^{2}}+\cdots
$$

For $\lambda_{1}$ large enough, the non-conserving terms with order higher than the first are strongly suppressed, and the LOA expectation value of a linear operator $\mathcal{O}$ can be extracted as $\mathcal{O}_{1}=\mathcal{O}_{0}+\Delta \mathcal{O}_{1}=\mathcal{O}_{0}\left(d_{0}\right)+\mathcal{O}\left(d_{0} \Pi\left[d_{0} d_{0}\right] d_{0}\right)=$ $\mathcal{O}_{0}\left(d_{0}\right)+\lambda_{1}\left[\mathcal{O}\left(D_{1}^{\lambda_{1}}\right)-\mathcal{O}\left(d_{0}\right)\right]$, where $D_{1}^{\lambda_{1}}$ denotes the first-order phonon LOA Green's function rescaled by $\lambda_{1}$ :

$$
D_{1}^{\lambda_{1}}=d_{0}+d_{0} \Pi_{1}^{\lambda_{1}} d_{0}, \Pi_{1}^{\lambda_{1}}=\frac{\Pi\left[d_{0} d_{0}\right]}{\lambda_{1}},
$$

To proceed with the computation of the second order LOA, we build the rescaled version $\Pi_{2}^{\lambda_{2}}$ of the phonon self-energy $\Pi\left[D_{1} D_{1}\right]$ :

$$
\begin{aligned}
\Pi_{2}^{\lambda_{2}} & =\frac{1}{\lambda_{2}} \Pi\left[D_{1}^{\lambda_{2}} D_{1}^{\lambda_{2}}\right] \\
& =\frac{1}{\lambda_{2}} \Pi\left[\left(d_{0}+\frac{1}{\lambda_{2}} \Delta d_{1}\right)\left(d_{0}+\frac{1}{\lambda_{2}} \Delta d_{1}\right)\right], \\
& =\frac{1}{\lambda_{2}} \Pi\left[d_{0} d_{0}\right]+\frac{1}{\lambda_{2}^{2}} \Pi\left[2 d_{0} \Delta d_{1}\right]+\frac{1}{\lambda_{2}^{3}} \Pi\left[\Delta d_{1} \Delta d_{1}\right]
\end{aligned}
$$

where $\Delta d_{1}=d_{0} \Pi_{1} d_{0}=d_{0} \Pi\left[d_{0} d_{0}\right] d_{0}$ and the linearity of $\Pi$ has been exploited. It can be checked that terms of order $i$ are divided by $\lambda_{2}^{i}$. This remains true for any polynomial in $\Pi_{2}^{\lambda_{2}}$, and thus for the rescaled Green's function $D_{2}^{\lambda_{2}}$ provided by the second SCBA iteration. Again, if the scaling factor $\lambda_{2}$ is appropriately chosen ${ }^{54}$, the (nonconserving) terms of order higher than the second are strongly suppressed, and the second-order LOA expectation value can be obtained as $\mathcal{O}_{2}=\mathcal{O}_{0}+\Delta \mathcal{O}_{1}+\Delta \mathcal{O}_{2}$, with $\Delta \mathcal{O}_{2}=\lambda_{2}^{2}\left[\mathcal{O}\left(D_{2}^{\lambda_{2}}\right)-\mathcal{O}\left(d_{0}\right)\right]-\lambda_{2} \Delta \mathcal{O}_{1}$.

This strategy can be generalized to the computation of the phonon Green's function LOA at any order. In particular, from a Green's function exact to the $(N-1)^{t h}$ order, rescaled by a factor $\lambda_{N}$

$$
D_{N-1}^{\lambda_{N}}=d_{0}+\frac{1}{\lambda_{N}} \Delta d_{1}+\cdots+\frac{1}{\lambda_{N}^{N-1}} \Delta d_{N-1}
$$

where $\Delta_{i}$ denotes the term of order $i$ of the Green's function perturbation series, we can obtain a rescaled self energy $\Pi_{N}^{\lambda_{N}}$ exact to the $N^{t h}$ order as 


$$
\Pi_{N}^{\lambda_{N}}=\Pi\left[D_{N-1}^{\lambda_{N}} D_{N-1}^{\lambda_{N}}\right]=\Pi\left[\frac{d_{0} d_{0}}{\lambda_{N}}+\cdots+\frac{\sum_{i=0}^{N-1} \Delta d_{i} \Delta d_{N-1-i}}{\lambda_{N}^{N}}+\frac{\sum_{i=0}^{N-2} \Delta d_{i+1} \Delta d_{N-1-i}}{\lambda_{N}^{N+1}}+\cdots\right] .
$$

In this paper, a conserving analytical continuation technique relying on the Padé approximation is used ${ }^{50,52}$. We compute the LOA expectation values of the thermal current up to the third order, as our results indicate that the Padé approximants $0 / 1,1 / 1$ and $1 / 2$ built from them can already provide good estimates of the SCBA converged current.

The rescaling technique has been presented for the case of the self-energy (6), which depends on the square of the phonon Green's function. However, it is easy to argue that it can be used exactly in the same form in the case of the composition $\Sigma\left[G^{k}\right]$ of a linear self-energy $\Sigma$ with any positive integer power $k$ of the Green's function $G$. By going a small step further, we observe that, due to the linearity of $\Sigma$, the method can be applied to selfenergies of the form $\Sigma\left[\sum_{i=0}^{M} f_{i} G^{k(i)}\right]=\sum_{i=0}^{M} f_{i} \Sigma\left[G^{k(i)}\right]$, where the function $k$ associates $i$ a positive integer. If the functional $\Sigma$ is bounded, the previous equality holds also for $M=\infty$, and, by taking $k(i)=i$, we can conclude that the rescaling technique is applicable to all the conserving self-energies of the form $\Sigma[f(G)]$, where $f$ is an analytic function.

These considerations show that the case of the phononphonon scattering self-energy on which the paper focuses, while important, represents just a very simple example among the wide range of physical situations the method can deal with. The achievable numerical efficiency will obviously depend on the particular case at hand.

\section{Richardson extrapolation}

When the anharmonic scattering is strong, as in the case of temperatures much higher than room temperature (see below, subsection III B), the convergence of the series of the Padé approximants can be slow. In such cases, we propose to apply the Richardson extrapolation to the LOA expectation values analytically continued by the Padé approximants $N-1 / N$.

The Richardson extrapolation is a method to accelerate the convergence of monotone series. If a sequence $S_{N}$ approaches monotonically a certain value $S$, we can write $S_{N}$ in an asymptotic form as ${ }^{56}$

$$
S_{N} \approx S+\frac{c_{1}}{N}+\frac{c_{2}}{N^{2}}+\frac{c_{3}}{N^{3}}+\cdots,
$$

where $c_{1}, c_{2}, \cdots$ are unknown parameters. By considering only the first correction term for $S_{N}$ and $S_{N+1}$

$$
S_{N}=S+\frac{c_{1}}{N}, \quad S_{N+1}=S+\frac{c_{1}}{N+1},
$$

the first-order Richardson extrapolation can be expressed as

$$
S_{N}^{[1]}=(N+1) S_{N+1}-N S_{N}
$$

We used this technique to accelerate the convergence of the sequence $\mathcal{O}_{N-1 / N}$ of the LOA expectation values of an operator $\mathcal{O}$, analytically continued by the Padé approximants $N-1 / N$. If, as $N \rightarrow \infty, \mathcal{O}_{N-1 / N}$ converges monotonically, we can set $S_{N}=\mathcal{O}_{N-1 / N}$ and apply Eq. (19). In the paper, the Richardson extrapolation is applied to the sequence elements $\mathcal{O}_{0 / 1}$ and $\mathcal{O}_{1 / 2}$ as

$$
S_{1}^{[1]}=2 S_{2}-S_{1}=2 \mathcal{O}_{1 / 2}-\mathcal{O}_{0 / 1},
$$

to obtain an improved estimate $S_{1}^{[1]}$ of the corresponding SCBA result.

\section{RESULTS AND DISCUSSIONS}

In this section, we present and discuss the simulation results for the thermal current in $\mathrm{Si}$ and Ge NWs, obtained by using either the LOA analytically continued with Padé approximants and possibly further improved by using the Richardson extrapolation or the SCBA. The SCBA results have been obtained within a tolerance of $1 \%$ on the current conservation. All the NWs are undoped. The resulting low electron density allows us to neglect the electron contribution to the thermal current and the electron-phonon scattering. In all the considered cases, the thermal current is sustained by a temperature gradient of $0.1 \mathrm{~K}$, enforced at the NW extremities.

In subsection III A, we consider the case of NWs with a uniform cross-section at room temperature. In subsection III B, we discuss the results obtained by increasing the temperature up to $900 \mathrm{~K}$. In these conditions, the increase in the phonon population entails a significant enhancement of the phonon-phonon scattering rate. Finally, as an example of geometry-driven mode-mixing enhancement, in subsection III C we address the case of NWs with discontinuous cross-section. 


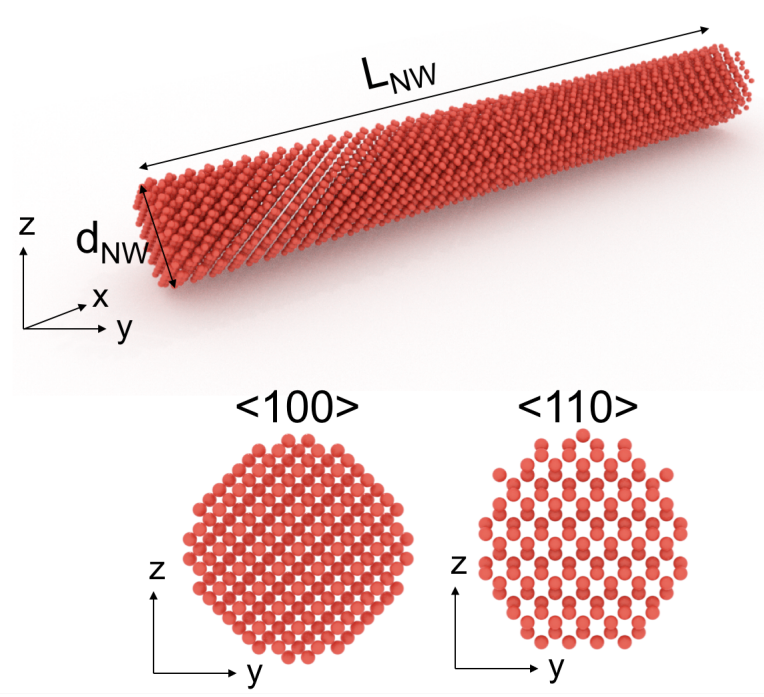

FIG. 2. Top panel: atomic structure of the $\langle 100\rangle$-oriented Si or Ge NWs investigated in subsection III A. The length and the diameter of the nanowires are $\mathrm{L}_{\mathrm{NW}}=60 \mathrm{~nm}$ and $\mathrm{d}_{\mathrm{NW}}=3$ $\mathrm{nm}$, respectively. Bottom panel: cross-section view of (left) the $\langle 100\rangle$-oriented and (right) the $\langle 110\rangle$-oriented nanowires.

\section{A. Uniform cross-section nanowires at room temperature}

The atomic structure of the considered Si and Ge NWs is depicted in Fig. 2. The nanowires are $60 \mathrm{~nm}$ long and have a circular cross-section with a diameter of 3 $\mathrm{nm}$. The Ge NW is oriented in the $\langle 110\rangle$ direction, while for the Si NW both the $\langle 110\rangle$ and $\langle 100\rangle$ directions are considered. In the following, we refer as "ballistic" the results for the thermal current obtained with uncoupled phonon modes, namely in the harmonic approximation.

Fig. 3 (a) illustrates the values of thermal current along the $\langle 110\rangle$-oriented $\mathrm{Si} \mathrm{NW}$, in the ballistic regime and in the presence of anharmonic phonon-phonon scattering. For this latter case, the SCBA converged value and the LOA approximations up to the third order are reported. The results confirm that the rescaling technique is actually able to provide current-conserving expectation values at any order. However, the LOA power series diverges, due to the already significant anharmonic scattering strength at room temperature ${ }^{25}$. Analogously to the case of the previously investigated electron-phonon selfenergy $^{52,54}$, an analytical continuation of the LOA series based on the Padé approximation has been resorted to obtain a convergent behavior. Fig. 3 (b) shows the Padé approximants built from the LOA values. The sequence of Padé approximants $0 / 1,1 / 1$ and $1 / 2$ oscillates around the SCBA value, the best approximation of which is obtained by the $1 / 2$ approximant with a relative error $\varepsilon$
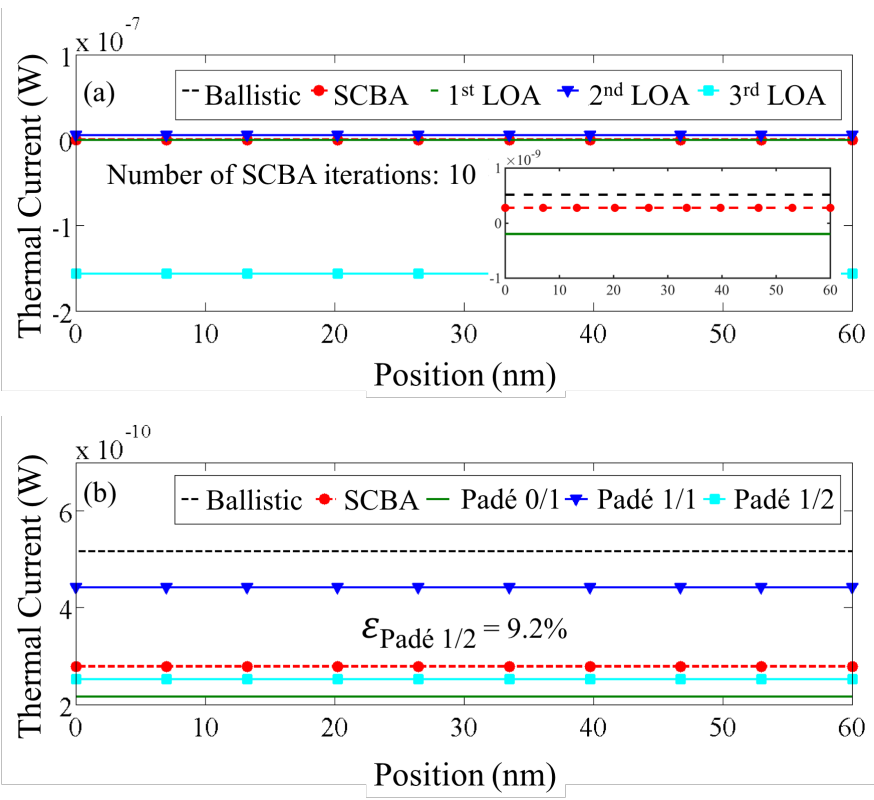

FIG. 3. (a) Room temperature ballistic, SCBA and LOA thermal currents up to the third order in the $\langle 110\rangle$-oriented Si NW. Inset: enlargement around the value of the SCBA current. (b) Padé approximants $0 / 1,1 / 1$ and $1 / 2$ built from the LOA results. The ballistic and SCBA currents are also represented.

$\left(=100 \times\left|\mathcal{I}_{\mathrm{SCBA}}-\mathcal{I}_{\text {Pade }}\right| / \mathcal{I}_{\mathrm{SCBA}}\right)$ lower than $9.2 \%$. This result is achieved with around half of the computational burden needed for the SCBA (6 against 10 SCBA iterations). The same behavior is observed for the $\langle 100\rangle$ oriented Si NW (not shown). In this case, the divergence of the LOA series is faster, which suggests stronger anharmonic interactions. The Padé approximant $1 / 2$ is also in this case in good agreement with the SCBA value $(\varepsilon=10 \%)$.

Figure 4 illustrates the corresponding results obtained for the $\langle 110\rangle$-oriented Ge NW. The divergence of the LOA series is slower than for the Si NWs, which indicates a higher ballisticity (the lower thermal conductivity of Ge NWs ${ }^{40,67-69}$ with respect to Si NWs is a consequence of the lower group velocity of the acoustic phonon branches ${ }^{25}$, which mainly sustain the thermal current). Figure 4 (b) shows that, once again, the 1/2 Padé approximant provides a good estimate of the SCBA current $(\varepsilon=10 \%)$, while resulting in an improvement of the numerical efficiency similar to that found for the Si NWs.

\section{B. Uniform cross-section nanowires at higher temperatures}

A different scenario is observed when temperatures much higher than $300 \mathrm{~K}$ are considered. Table I reports 

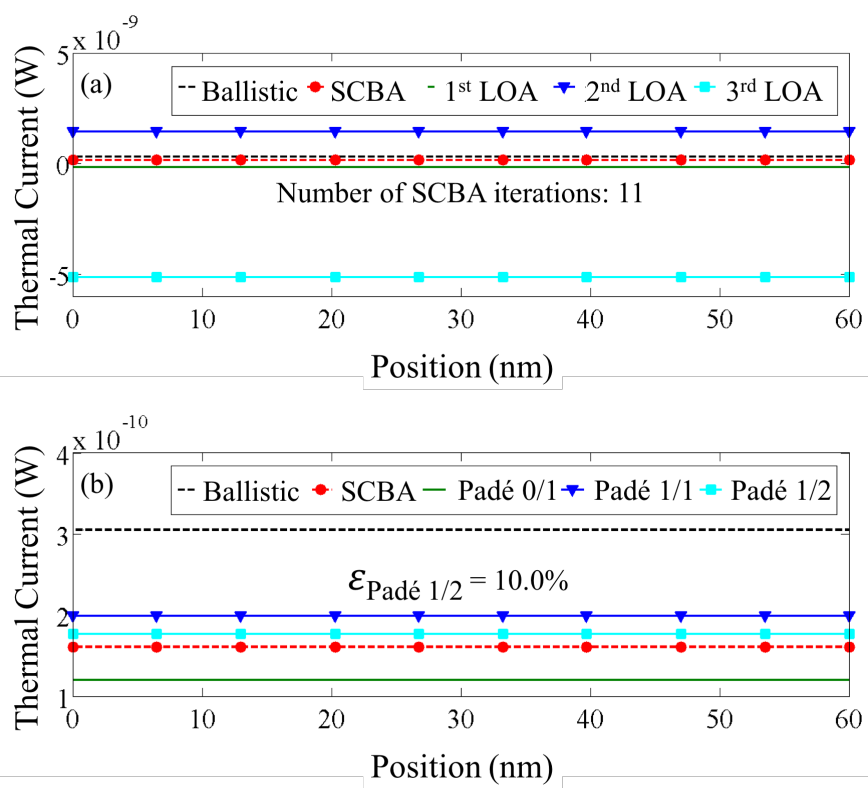

FIG. 4. (a) Room temperature ballistic, SCBA and LOA thermal currents up to the third order in the $\langle 110\rangle$-oriented Ge NW. (b) Padé approximants $0 / 1,1 / 1$ and $1 / 2$ built from the LOA results. The ballistic and SCBA currents are also represented.

the LOA thermal current analytically continued by the $0 / 1$ and $1 / 2$ Padé approximants for the $\langle 110\rangle$-oriented $\mathrm{Si}$ NW considered in subsection III A, and the corresponding values obtained through a first-order Richardson extrapolation. The SCBA and ballistic current, and the number of SCBA iterations needed to obtain each result are also shown. The data are provided for temperatures ranging from $300 \mathrm{~K}$ to $900 \mathrm{~K}$. It can be noticed that the current degradation with respect to the ballistic case $\delta \mathcal{I}=100 \times\left[1-\mathcal{I}_{\mathrm{SCBA}} / \mathcal{I}_{\text {bal }}\right]$, where $\mathcal{I}_{\mathrm{SCBA}}$ and $\mathcal{I}_{\text {bal }}$ are the SCBA and ballistic value of the current, respectively, significantly increases with the temperature: $\delta \mathcal{I}=46 \%$ at $300 \mathrm{~K}, 64 \%$ at $500 \mathrm{~K}, 74 \%$ at $700 \mathrm{~K}$ and $81 \%$ at $900 \mathrm{~K}$. This is a consequence of the enhancement of the anharmonic interactions at high temperature ${ }^{25}$. The accuracy of the Padé approximant $1 / 2$ in retrieving the SCBA result progressively degrades with increasing temperature $(\varepsilon=17.4 \%$ at $500 \mathrm{~K}, 20.8 \%$ at $700 \mathrm{~K}$, and $29.2 \%$ at 900 $\mathrm{K})$, suggesting that higher-order LOA terms are needed. However, it can be seen that in all the cases the Richardson extrapolation obtained from the Padé approximants $0 / 1$ and $1 / 2$ significantly improves the estimate of the SCBA result (we find $\varepsilon=4.0 \%$ at $300 \mathrm{~K}, 0.0 \%$ at 500 $\mathrm{K}, 1.3 \%$ at $700 \mathrm{~K}$, and $11.3 \%$ at $900 \mathrm{~K})$. We remark that the use of the Richardson extrapolation does not require further iterations and therefore does not increase the computational burden. In this way, it is possible to considerably boost the numerical efficiency of the LOA
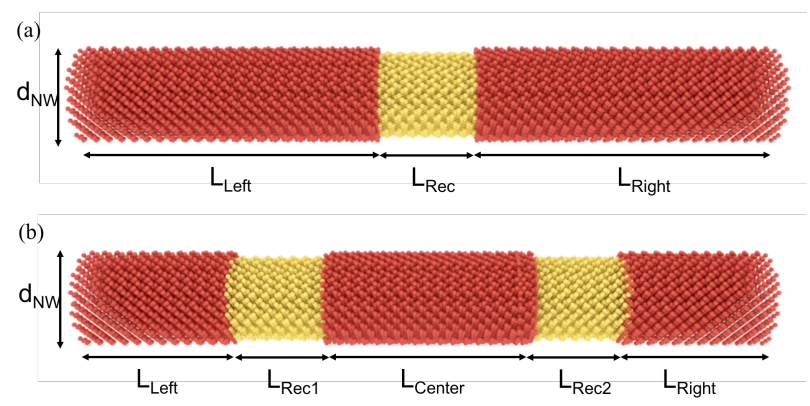

FIG. 5. (a) Illustration of the atomic structure (not to scale) of the $\langle 100\rangle$-oriented Si NW in the presence of a single recessed region (NWR1). (b) Illustration of the atomic structure (not to scale) of the $\langle 100\rangle$-oriented $\mathrm{Si} \mathrm{NW}$ in the presence of two recessed regions (NWR2). For the sake of clarity, the Si atoms in the recessed regions are shown in yellow.

with respect to the SCBA scheme. For example, Table I shows that approximating the anharmonic thermal current at $\mathrm{T}=900 \mathrm{~K}$ within a relative error close to $10 \%$ is more than 7 times faster than computing the corresponding SCBA result.

We tested the first-order Richardson extrapolation also in the case of the self-energies describing electron-phonon interactions considered in Ref. 54. In all the cases in which the sequence of the $N-1 / N$ Padé approximants converged monotonically, we again obtained a significant acceleration.

\section{Nanowires with discontinuous cross-section}

The atomic structure of the NWs with discontinuous cross-section is sketched in Fig. 5. We consider a $\langle 100\rangle$ oriented Si NW with $\mathrm{L}_{\mathrm{NW}}=60 \mathrm{~nm}$ and $\mathrm{d}_{\mathrm{NW}}=4 \mathrm{~nm}$ at room temperature. The choice of the $\langle 100\rangle$ orientation allows us to discuss a different convergence behavior, which we did not observe in the $\langle 110\rangle$ orientation. The NW in Fig. 5 (a), which we will refer to as NWR1, has one recessed region of length $\mathrm{L}_{\mathrm{Rec}}=10 \mathrm{~nm}$. The recess is obtained by removing the outermost layer of atoms. The length of the left/right unrecessed parts is $\mathrm{L}_{\text {Left }}=\mathrm{L}_{\text {Right }}=25 \mathrm{~nm}$. The NW in Fig. 5 (b), which we will refer to as NWR2, has two recessed regions of length $\mathrm{L}_{\text {Rec1 }}=\mathrm{L}_{\mathrm{Rec} 2}=5 \mathrm{~nm}$. The lengths of the left, center, and right unrecessed regions are $\mathrm{L}_{\text {Left }}=12.5 \mathrm{~nm}$, $\mathrm{L}_{\text {Center }}=25 \mathrm{~nm}$, and $\mathrm{L}_{\text {Right }}=12.5 \mathrm{~nm}$, respectively.

These structures can be considered as elemental cells from which one-dimensional nanoscale phononic crystals with tailored phonon band structures ${ }^{70}$ could be assembled. We remark that, in our model, the discontinuities of the NW cross-section affect the self-energy in a qualitatively different way with respect to an increase of the temperature. This latter induces an increase of the anhar- 
TABLE I. LOA thermal currents analytically continued by the Padé approximants $0 / 1$ and $1 / 2$ and the corresponding Richardson extrapolations for the $\langle 110\rangle$-oriented Si NW, at different temperatures. The number of SCBA iterations required to obtain each approximation, the relative difference with respect to the SCBA results, and the corresponding ballistic and SCBA currents are also reported.

\begin{tabular}{|c|c|c|c|c|c|c|}
\hline & Ballistic & $1^{\text {st }} \mathrm{LOA}+$ Padé $0 / 1$ & $3^{r d} \mathrm{LOA}+$ Padé $1 / 2$ & $1^{1^{s t} \text { Richardson }}$ & $\overline{\text { SCBA }}$ & Temperature \\
\hline $\mathcal{I}_{p h}[\mathrm{~W}]$ & $5.16 \mathrm{e}-10$ & $2.17 \mathrm{e}-10$ & $2.53 \mathrm{e}-10$ & $2.89 \mathrm{e}-10$ & $2.78 \mathrm{e}-10$ & $300 \mathrm{~K}$ \\
\hline$\varepsilon[\%]$ & 85.6 & 21.9 & 9.0 & 4.0 & 0.0 & \\
\hline Iterations & 0 & 1 & 6 & 6 & 10 & \\
\hline $\mathcal{I}_{p h}[\mathrm{~W}]$ & $5.71 \mathrm{e}-10$ & $1.34 \mathrm{e}-10$ & $1.71 \mathrm{e}-10$ & $2.07 \mathrm{e}-10$ & $2.07 \mathrm{e}-10$ & $500 \mathrm{~K}$ \\
\hline$\varepsilon[\%]$ & 175.8 & 35.3 & 17.4 & 0.0 & 0.0 & \\
\hline Iterations & 0 & 1 & 6 & 6 & 19 & \\
\hline $\mathcal{I}_{p h}[\mathrm{~W}]$ & $5.89 \mathrm{e}-10$ & $8.87 \mathrm{e}-11$ & $1.22 \mathrm{e}-10$ & $1.56 \mathrm{e}-10$ & $1.54 \mathrm{e}-10$ & $700 \mathrm{~K}$ \\
\hline$\varepsilon[\%]$ & 282.5 & 42.4 & 20.8 & 1.3 & 0.0 & \\
\hline Iterations & 0 & 1 & 6 & 6 & 28 & \\
\hline $\mathcal{I}_{p h}[\mathrm{~W}]$ & $5.97 \mathrm{e}-10$ & $6.05 \mathrm{e}-11$ & $8.14 \mathrm{e}-11$ & $1.02 \mathrm{e}-10$ & $1.15 \mathrm{e}-10$ & $900 \mathrm{~K}$ \\
\hline$\varepsilon[\%]$ & 419.1 & 47.4 & 29.2 & 11.3 & 0.0 & \\
\hline Iterations & 0 & 1 & 6 & 6 & 44 & \\
\hline
\end{tabular}
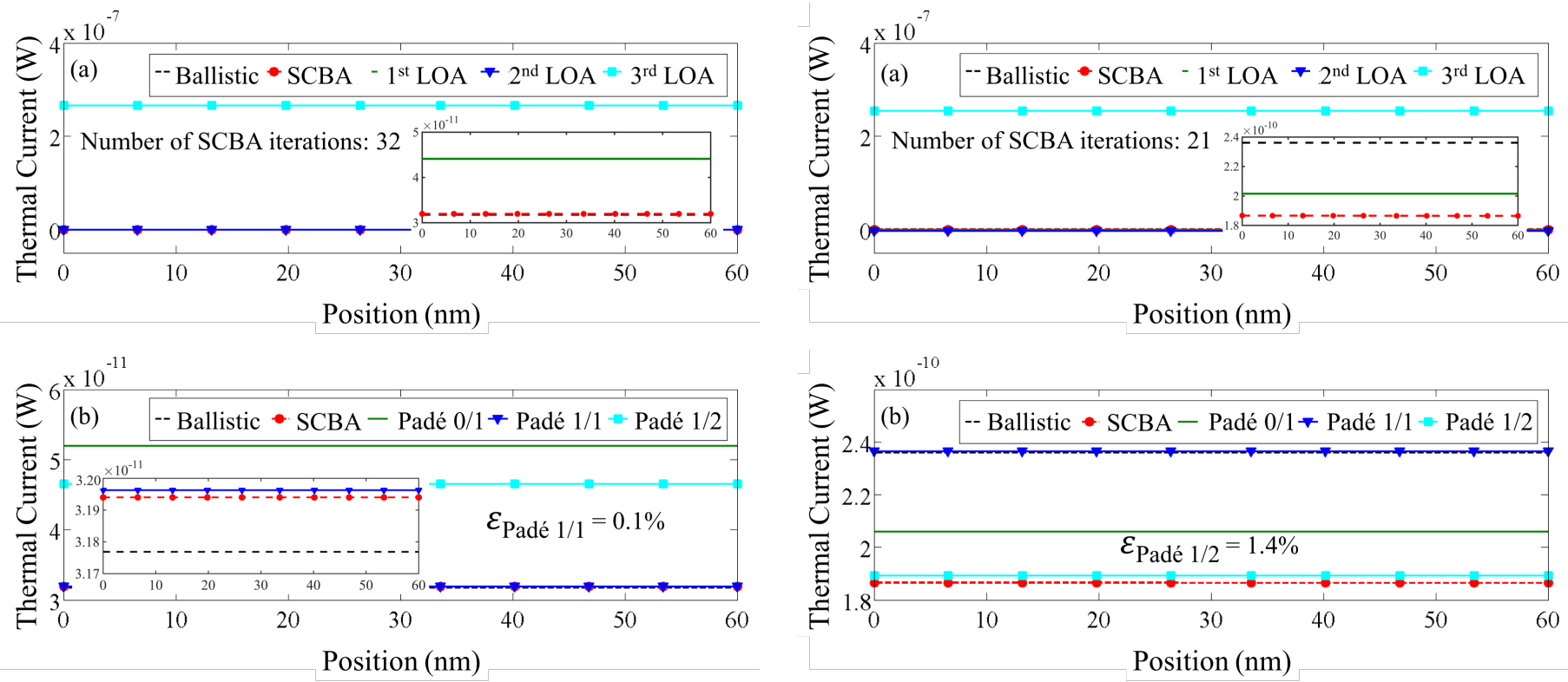

FIG. 6. (a) Room temperature ballistic, SCBA and LOA thermal currents up to the third order in the NWR1 structure. Inset: enlargement around the value of the SCBA current. (b) Padé approximants $0 / 1,1 / 1$ and $1 / 2$ built from the LOA results. The ballistic and SCBA currents are also represented. Inset: enlargement around the value of the SCBA current.

monic scattering rate essentially by modifying the noninteracting phonon Green's function. Differently, in the case of geometrical irregularities, this increase is mainly due to a change in the interaction strength, through the derivatives of the anharmonic components of the potential energy (see Eq. (5)).

Figure 6 illustrates the results obtained for the structure NWR1. Contrary to the cases previously discussed
FIG. 7. (a) Room temperature ballistic, SCBA and LOA thermal currents up to the third order in the NWR2 structure. Inset: enlargement around the value of the SCBA current. (b) Padé approximants $0 / 1,1 / 1$ and $1 / 2$ built from the LOA results. The ballistic and SCBA currents are also represented.

in subsections III A and IIIB, it can be noticed that the SCBA and the first-order LOA thermal currents are higher than the ballistic one. This behavior is similar to the one observed in the n-type $\langle 111\rangle$-oriented Si NWs in the case of electron-phonon scattering (see Table I in Ref. 54). Physically, the increase of the current in the presence of anharmonic scattering originates from the occurrence, in ballistic conditions, of localized phonon modes, induced by the cross-section nonuniformity. In 
the presence of mode-mixing, these modes can contribute to transport, therefore entailing an increase of the current. Figure 6 (b) shows that the best approximation of the SCBA current is provided by the 1/1 Padé approximant, able to almost perfectly recover the SCBA value $(\varepsilon=0.1 \%)$. This could suggest that in similar cases the sequence of $N / N$ Padé approximants converges faster than the $N-1 / N$ one. The LOA approximation turns out to be one order of magnitude faster, as only 3 SCBA iterations are needed to compute the 1/1 Padé approximant, against the 32 iterations required to obtain the converged SCBA result. This behavior is of course not always observed for NWs with surface recesses. Figure 7 shows the results for the slightly more complex NWR2 structure. In this case, the anharmonic scattering induces a decrease of the thermal current, and the behavior is the same as that observed for NWs with uniform cross-section. Accordingly, the best approximation of the SCBA thermal current is provided by the $1 / 2$ Padé approximant. The relative error with respect to the SCBA result is $\varepsilon=1.4 \%$, and the numerical efficiency is improved by more than 3 times.

It is interesting to compare the results for the structure NWR1 with those obtained for the NW with uniform cross-section at $\mathrm{T}=700 \mathrm{~K}$. We observe that, while the total number of SCBA iterations slightly increase from 28 to 32 , the relative error of the best Padé approximant with respect to the SCBA significantly improves from $20.8 \%$ to $1 \%$. The different behavior can be attributed to the local character of the NW cross-section discontinuities. A localized scattering center is less efficient in triggering high order scattering processes with respect to a homogeneous increase of the temperature. This explains the higher accuracy of the analytically continued LOA in the case of the NWR1 structure. On the other hand, within the SCBA scheme, localization effects typically result in significant spatially localized fluctuations of the current, which need numerous iterations to be canceled out. The SCBA scheme cannot benefit from the same improvement as the LOA, because its numerical efficiency is limited by the non-automatic current conservation.

\section{CONCLUSION}

We investigated the opportunity to adopt the LOA approach as a more efficient option with respect to the SCBA in the description of the anharmonic phononphonon scattering in thermal transport NEGF simulations. We found that the LOA, in the rescalingtechnique-based implementation, is able to provide the correct thermal current-conserving expectation values at any order. This method can be generalized to any conserving self-energy that analytically depends on the Green's function. We benchmarked the numerical efficiency of the LOA approach against the SCBA through atomistic phonon transport simulations in $\mathrm{Si}$ and $\mathrm{Ge}$ NWs. For constant cross-section NWs at room temperature, our results indicate that the Padé approximants built with the LOA currents up to the third order can provide high accuracy and a significant computational advantage with respect to the SCBA (typically, a reduction of the numerical burden by a factor 2). The largest improvement (up to a factor 10) of the computational efficiency relative to the SCBA has been observed in NWs with discontinuous cross-section. A similar improvement can be obtained also at temperatures substantially higher than the room temperature, if the first-order Richardson extrapolation is applied to the Padé $N-1 / N$ sequence in order to accelerate the convergence.

\section{ACKNOWLEDGEMENT}

This work was granted access to the HPC resources of Aix-Marseille Université financed by the project Equip@Meso (ANR-10-EQPX-29-01) and funded by the NOODLES contract (ANR-13-NANO-0009) of the ANRFrench National Research Agency. The author Y. Lee would like to thank the AMIDEX for financial support.
* youseung.lee@iis.ee.ethz.ch

$\dagger$ marc.bescond@iis.u-tokyo.ac.jp

${ }^{1}$ K. S. Novoselov, A. K. Geim, S. V. Morozov, D. Jiang, Y. Zhang, S. V. Dubonos, I. V. Grigorieva, and A. A. Firsov, Science 306, 666 (2004).

${ }^{2}$ L. Bartels, G. Meyer, and K.-H. Rieder, Phys. Rev. Lett. 79, 697 (1997).

3 A. I. Yanson, G. R. Bollinger, H. E. van den Brom, N. Agrat, and J. M. van Ruitenbeek, Nature 395, 783 (1998).

4 J. A. Stroscio and R. J. Celotta, Science 306, 242 (2004).

5 J. J. Pla, K. Y. Tan, J. P. Dehollain, W. H. Lim, J. J. L. Morton, D. N. Jamieson, A. S. Dzurak, and A. Morello, Nature 489, 541 (2012).
${ }^{6}$ S. D. Suk et al., in IEEE International Electron Devices Meeting, 2005. IEDM Technical Digest., Washington, DC, 2005 (2005) pp. 717-720.

7 H. Mertens et al., in 2016 IEEE Symposium on VLSI Technology (2016) pp. 1-2.

${ }^{8}$ H. Schmid, C. Bessire, M. T. Bjrk, A. Schenk, , and H. Riel, Nano Lett. 12, 699 (2012).

9 M. Law, L. E. Greene, J. C. Johnson, R. Saykally, and P. Yang, Nat. Mater. 4, 455 (2005).

10 T. J. Kempa, B. Tian, D. R. Kim, J. Hu, X. Zheng, and C. M. Lieber, Nano Lett. 8, 3456 (2008).

11 A. I. Hochbaum, R. Chen, R. D. Delgado, W. Liang, E. C. Garnett, M. Najarian, A. Majumdar, and P. Yang, Nature 451, 163 (2008). 
12 A. I. Boukai, Y. Bunimovich, J. Tahir-Kheli, J.-K. Yu, W. A. G. III, and J. R. Heath, Nature 451, 168 (2008).

13 G. D. Mahan, Many-Particle Physics (Plenum, New York, 1990).

14 S. Datta, Electronic Transport in Mesoscopic Systems (Cambridge University Press, Cambridge, U.K., 1995).

15 H. Haug and A.-P. Jauho, Quantum Kinetics in Transport and Optics of Semiconductors, vol. 123 of Springer Series in Solid-State Sciences (Springer, Berlin, New York, 1996).

16 D. K. Ferry and S. M. Goodnick, Transport in Nanostructures (Cambridge University Press, Cambridge, U.K., 1997).

17 X. Zhao, C. M. Wei, L. Yang, and M. Y. Chou, Phys. Rev. Lett. 92, 236805 (2004).

18 M. Luisier and G. Klimeck, Phys. Rev. B 80, 155430 (2009).

19 Y.-M. Niquet, H. Mera, and C. Delerue, Appl. Phys. Lett. 100, 153119 (2012).

20 N. Mingo and L. Yang, Phys. Rev. B 68, 245406 (2003).

21 Y. Xu, J.-S. Wang, W. Duan, B.-L. Gu, and B. Li, Phys. Rev. B 78, 224303 (2008).

22 P. Martin, Z. Aksamija, E. Pop, and U. Ravaioli, Phys. Rev. Lett. 102, 125503 (2009).

23 T. Markussen, A.-P. Jauho, and M. Brandbyge, Phys. Rev. B 79, 035415 (2009).

24 M. Luisier, J. Appl. Phys. 110, 074510 (2011).

25 M. Luisier, Phys. Rev. B 86, 245407 (2012).

${ }^{26}$ R. Rhyner and M. Luisier, Nano Lett. 16, 1022 (2016).

27 J. Srinivasan, S. V. Adve, P. Bose, and J. A. Rivers, in International Conference on Dependable Systems and Networks, 2004 (2004) pp. 177-186.

${ }^{28}$ L. D. Hicks and M. S. Dresselhaus, Phys. Rev. B 47, 16631(R) (1993).

29 A. L. Moore, S. K. Saha, R. S. Prasher, and L. Shi, Appl. Phys. Lett. 93, 083112 (2008).

30 S. P. Hepplestone and G. P. Srivastava, Phys. Rev. B 82, 144303 (2010).

31 Z. Aksamija and I. Knezevic, Phys. Rev. B 82, 045319 (2010).

32 G. Romano, K. Esfarjani, D. A. Strubbe, D. Broido, and A. M. Kolpak, Phys. Rev. B 93, 035408 (2016).

33 S. G. Volz and G. Chen, Phys. Rev. B 61, 2651 (2000).

${ }^{34}$ W. G. Hoover and C. G. Hoover, Condens. Matter Phys. 8, 247 (2005).

35 S. chuang Wang, X. gang Liang, X. hua Xu, and T. Ohara, J. Appl. Phys. 105, 014316 (2009).

36 D. Donadio and G. Galli, Nano Lett. 10, 847 (2010).

37 Y. He and G. Galli, Phys. Rev. Lett. 108, 215901 (2012).

38 S. Narasimhan and D. Vanderbilt, Phys. Rev. B 43, 4541(R) (1991).

39 A. Ward, D. A. Broido, D. A. Stewart, and G. Deinzer, Phys. Rev. B 80, 125203 (2009).

40 J. Garg, N. Bonini, B. Kozinsky, and N. Marzari, Phys. Rev. Lett. 106, 045901 (2011).

41 N. Mingo, Phys. Rev. B 74, 125402 (2006).

${ }^{42}$ M. Galperin, A. Nitzan, and M. A. Ratner, Phys. Rev. B 75, 155312 (2007).
43 J. M. Luttinger and J. C. Ward, Phys. Rev. 118, 1417 (1960).

44 J. M. Luttinger, Phys. Rev. 119, 1153 (1960).

45 G. Baym and L. P. Kadanoff, Phys. Rev. 124, 287 (1961).

46 G. Baym, Phys. Rev. 127, 1391 (1962).

47 T. Frederiksen, M. Brandbyge, N. Lorente, and A.-P. Jauho, Phys. Rev. Lett. 93, 256601 (2004).

48 M. Paulsson, T. Frederiksen, and M. Brandbyge, Phys. Rev. B 72, 201101(R) (2005).

49 H. Mera, M. Lannoo, C. Li, N. Cavassilas, and M. Bescond, Phys. Rev. B 86, 161404(R) (2012).

50 H. Mera, M. Lannoo, N. Cavassilas, and M. Bescond, Phys. Rev. B 88, 075147 (2013).

51 M. Bescond, C. Li, H. Mera, N. Cavassilas, and M. Lannoo, J. Appl. Phys. 114, 153712 (2013).

52 Y. Lee, M. Lannoo, N. Cavassilas, M. Luisier, and M. Bescond, Phys. Rev. B 93, 205411 (2016).

${ }^{53}$ H. Mera, T. G. Pedersen, and B. K. Nikolić, Phys. Rev. B 94, 165429 (2016).

54 Y. Lee, M. Bescond, N. Cavassilas, D. Logoteta, L. Raymond, M. Lannoo, and M. Luisier, Phys. Rev. B 95, 201412(R) (2017).

55 L. F. Richardson and J. A. Gaunt, Philosophical Transactions of the Royal Society of London A: Mathematical, Physical and Engineering Sciences 226, 299 (1927), http://rsta.royalsocietypublishing.org/content/226/636646/299.full.pdf.

56 C. M. Bender and S. A. Orszag, Advanced Mathematical Methods for Scientists and Engineers I: Asymptotic Methods and Perturbation Theory (Springer, New York, 1999).

57 C. Brezinski, J. Comput. Appl. Math. 122, 1 (2000).

58 Z. Sui and I. P. Herman, Phys. Rev. B 48, 17938 (1993).

59 A. Paul, M. Luisier, and G. Klimeck, J. Comput. Electron. 9, 160 (2010).

60 M. Luisier, A. Schenk, and W. Fichtner, Phys. Rev. B 74, 205323 (2006).

61 M. Cardona and T. Ruf, Solid State Communications 117, 201 (2001)

62 G. Stefanucci and R. van Leeuwen, Nonequilibrium ManyBody Theory of Quantum Systems (Cambridge University Press, Cambridge, U.K., 2013).

63 M. P. Anantram, M. S. Lundstrom, and D. E. Nikonov, Proceedings of the IEEE 96, 1511 (2008).

64 A. Svizhenko and M. P. Anantram, IEEE Trans. Electron Devices 50, 1459 (2003).

65 S. Jin, Y. J. Park, and H. S. Min, J. Appl. phys. 99, 123719 (2006).

66 M. Frey, A. Esposito, and A. Schenk, in Proceedings of the 38th European Solid-State Device Research Conference (ESSDERC), Edinburgh, UK (IEEE, Piscataway, NJ, 2008) (2008) p. 258.

67 C. J. Glassbrenner and G. A. Slack, Phys. Rev. 134, A1058 (1964).

68 S. Sarikurt, A. Ozden, A. Kandemir, C. Sevik, A. Kinaci, J. B. Haskins, and T. Cagin, J. Appl. Phys. 119, 155101 (2016).

69 T. Feng and X. Ruan, Phys. Rev. B 93, 045202 (2016).

70 A. Khelif and A. Adibi, Phononic Crystals: Fundamentals and Applications (Springer-Verlag, New York, 2015). 